\title{
Tunable near- to mid-infrared pump terahertz probe spectroscopy in reflection geometry
}

\author{
S. J. Zhang ${ }^{1}$, Z. X. Wang ${ }^{1}$, T. Dong ${ }^{1, \dagger}$, N. L. Wang ${ }^{1,2, \ddagger}$ \\ ${ }^{1}$ International Center for Quantum Materials, School of Physics, Peking University, Beijing 100871, China \\ ${ }^{2}$ Collaborative Innovation Center of Quantum Matter, Beijing 100871, China \\ Corresponding authors. E-mail: † taodong@pku.edu.cn, ${ }^{\ddagger}$ nlwang@pku.edu.cn \\ Received June 29, 2017; accepted July 24, 2017
}

\begin{abstract}
Strong-field mid-infrared pump-terahertz ( $\mathrm{THz}$ ) probe spectroscopy has been proven as a powerful tool for light control of different orders in strongly correlated materials. We report the construction of an ultrafast broadband infrared pump- $\mathrm{THz}$ probe system in reflection geometry. A two-output optical parametric amplifier is used for generating mid-infrared pulses with GaSe as the nonlinear crystal. The setup is capable of pumping bulk materials at wavelengths ranging from $1.2 \mu \mathrm{m}$ to $15 \mu \mathrm{m}$ and beyond, and detecting the subtle, transient photoinduced changes in the reflected electric field of the $\mathrm{THz}$ probe at different temperatures. As a demonstration, we present $15 \mu \mathrm{m}$ pump-THz probe measurements of a bulk $\mathrm{EuSbTe}_{3}$ single crystal. A $0.5 \%$ transient change in the reflected $\mathrm{THz}$ electric field can be clearly resolved. The widely tuned pumping energy could be used in mode-selective excitation experiments and applied to many strongly correlated electron systems.
\end{abstract}

Keywords mid-infrared pump, terahertz spectroscopy

PACS numbers 78.47.jg, 78.47.D-

Over the past few decades, ultrafast spectroscopic techniques have provided new insights into exciting collective and single-particle modes of quantum materials and to track their subsequent decay pathways back to the equilibrium state. Most ultrafast experiments performed to date used near-infrared (NIR) and visible pulses, which are more accessible than mid-infrared (MIR) or far-infrared pulses, as most commercial ultrafast lasers are designed to generate narrowband pulses in the NIR range. In strongly correlated materials, in addition to the highly nonthermal electron distribution excited by high energy $(\sim \mathrm{eV})$ photons $[1,2]$, optical excitation can also result in a variety of novel dynamical phenomena, such as photoinduced insulator-to-metal transitions by "photodoping" Mott insulators [3] and optical melting of different orders $[4,5]$.

Low-energy excitations in strongly correlated materials, such as superconducting energy gap, Josephson plasma resonances, or specific lattice vibrations, always extend from gigahertz to MIR frequencies [11]. Thanks to recent developments in the generation of subpicosecond-duration laser pulses ranging from MIR

\footnotetext{
*arXiv: 1708.01991.
}

to terahertz $(\mathrm{THz})$ frequencies [6], low-energy excitations can be selectively controlled without delivering excess energy to other excitation pathways [7-10]. Among such experiments, the most exciting one may be the observation of transient superconductivity in $\mathrm{YBa}_{2} \mathrm{Cu}_{3} \mathrm{O}_{6.5}$ at room temperature $[9,12]$, in which the Josephson plasma edges formed by superfluid carriers in $\mathrm{YBa}_{2} \mathrm{Cu}_{3} \mathrm{O}_{6.5}$ after excitation with $15 \mu \mathrm{m}$ pulses were detected in a $c$-axis time-domain $\mathrm{THz}$ measurement. Ultrafast broadband infrared pump- $\mathrm{THz}$ probe spectroscopy has been proven as a powerful tool for manipulating and detecting different orders in strongly correlated materials.

Here we report the construction of an ultrafast optical system capable of pumping a bulk sample from NIR to MIR frequencies (wavelengths of $1.2-15 \mu \mathrm{m}$ and even beyond) and probing at $\mathrm{THz}$ frequencies $(\sim 0.25-2.5 \mathrm{THz})$ in reflection geometry. We demonstrate the ability of this system to interrogate low-energy excitations in materials by photoexciting a bulk $\mathrm{EuSbTe}_{3}$ crystal at room temperature using $15 \mu \mathrm{m}$ pulses and probing it with $\mathrm{THz}$ pulses, enabling us to time-resolve the photoinduced change in the $\mathrm{THz}$ electric field.

Figure 1 shows a diagram depicting the basic design of our optical system, which starts with an amplified 


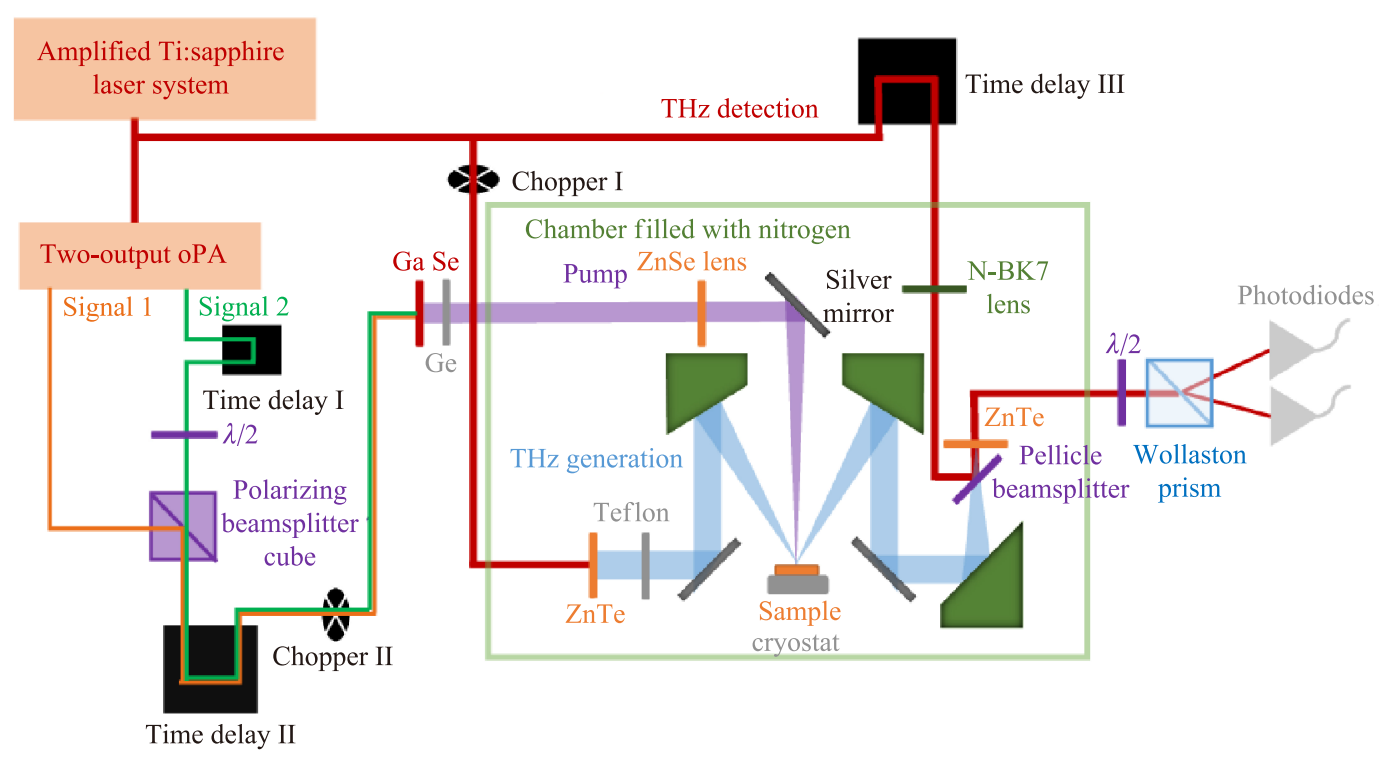

Fig. 1 Experimental schematic for ultrafast broadband infrared pump-THz probe spectroscopy. The pump pulses enter the nitrogen chamber via a $\mathrm{KBr}$ window and are focused onto the sample at normal incidence. THz pulses are generated and detected in the chamber; $800 \mathrm{~nm}$ pulses, which enter the chamber via quartz windows, are used to generate THz pulses by optical rectification and detect the electric field of reflected $\mathrm{THz}$ by electro-optic sampling. THz pulses are focused onto the sample at $30^{\circ}$ incidence while the sample sits at the end of a cold finger in a cryostat.

Ti:sapphire laser system producing $800 \mathrm{~nm}, 35$ fs pulses at a $1 \mathrm{kHz}$ repetition rate from a regeneration amplifier. The system has three arms that are used for the pump, $\mathrm{THz}$ generation, and $\mathrm{THz}$ detection.

A two-output optical parametric amplifier (OPA) is used for pump pulse generation. The two outputs both contain signal and idler beams ranging from 1.2 to 1.6 $\mu \mathrm{m}$ and 1.6 to $2.6 \mu \mathrm{m}$, respectively. Both the signal and idler beams can be used as the NIR pump in spectroscopy without focalization because they have sufficiently high fluences. To obtain the MIR pump, two signal beams that have been tuned to perpendicular polarization are used for difference frequency generation (DFG) collinearly on a 1-mm-thick z-cut GaSe crystal [13]. By tuning the frequency of the two signal beams and the orientation of the GaSe crystal to meet the type I or type II phase-matching condition, MIR pulses with tunable polarization ranging from 3 to $15 \mu \mathrm{m}$ can be generated $[14,15]$. A 5-mm-thick germanium plate used for blocking the two signal pulses is placed after the GaSe crystal. Pump pulses of $15 \mu \mathrm{m}$, where the available pump power is minimum in the current measurement, carry up to $2.5 \mu \mathrm{J}$ per pulse. A ZnSe convex lens is used to focus the pump pulses generated by DFG to obtain sufficient pump fluence.

The two signal beams used for DFG are seeded by the same white light continuum. So the generated MIR pulses are intrinsically carrier envelope phase stable, and their electric fields can be measured via electro-optic sampling (EOS) [16] with 35 fs gate pulses focused on a $100-\mu m$-thick z-cut GaSe crystal $[17,18]$. To obtain MIR pulses centered at $15 \mu \mathrm{m}$, the two signals of the OPA should be set at the frequencies shown in the top left panel of Fig. 2, which are measured with a grating
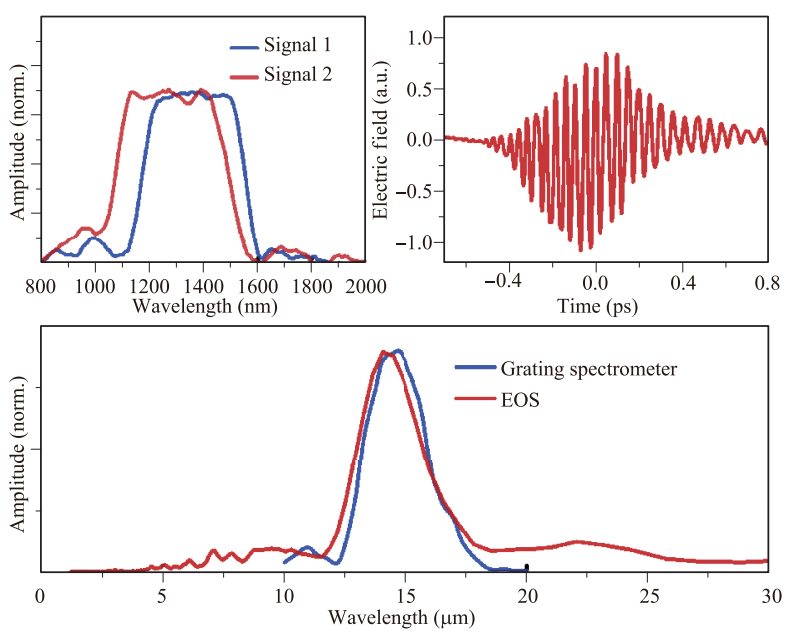

Fig. 2 Top left: Frequency spectra of two signal pulses obtained by a grating spectrometer. Top right: Time domain electric field of MIR pulses generated via DFG and measured via EOS in air. Bottom: Frequency spectra of MIR pulses obtained by EOS and a grating spectrometer. 
spectrometer. The time-domain electric field of the 15 $\mu \mathrm{m}$ pulses generated via DFG are measured via EOS in air, as shown in the top right panel of Fig. 2. The frequency-domain spectrum of the difference frequency pulses is acquired by Fourier transformation of the EOS signal, which is shown in the bottom panel of Fig. 2. We also measure the spectrum of the difference frequency pulse directly with a grating spectrometer equipped with a liquid-nitrogen-cooled mercury cadmium telluride detector. The result is also presented in the bottom panel of Fig. 2 for comparison. The two different measurements show very good agreement. The subtle differences between the two frequency spectra may result from the large susceptibilities to the orientation of the GaSe crystal and the non-negligible bandwidth of the two signal pulses.

The THz probe pulses are generated from pulses of 800 $\mathrm{nm}$ light whose polarization can be tuned by a $\lambda / 2$-plate using a 1-mm-thick (110) ZnTe crystal [19] without focalization. By using a $30^{\circ}$ off-axis parabolic mirror whose focal length is $54.45 \mathrm{~mm}$, we obtain a $0.63 \mathrm{~mm} \mathrm{THz} \mathrm{spot}$ size, as measured with a knife-edge scan. This rather small $\mathrm{THz}$ spot allows us to focus the pump beam into a spot less than $0.7 \mathrm{~mm}$ in size, resulting in generation of a higher MIR pump fluence. The $\mathrm{THz}$ profile was detected via EOS using 1-mm-thick ZnTe as the nonlinear detection crystal [20]. The THz pulses and $800 \mathrm{~nm}$ sampling pulses are focused onto the ZnTe crystal at normal incidence using a $90^{\circ}$ off-axis parabolic mirror and an N-BK7 convex lens, respectively. Figure 3 shows the time-dependent electric field of the $\mathrm{THz}$ pulse measured in air and nitrogen and the corresponding spectra as a function of frequency, obtained via Fourier transformation, of a test sample of $\mathrm{EuSbTe}_{3}$ [21] at room temperature. The dips in the Fourier amplitude spectra can be attributed to water absorption in air, and their positions in the frequency domain agree well with previously reported results $[22,23]$. The water absorption could be eliminated by either evacuating the chamber in which the $\mathrm{THz}$ beam is generated or filling the chamber with dry nitrogen gas. Because some mirrors in the chamber
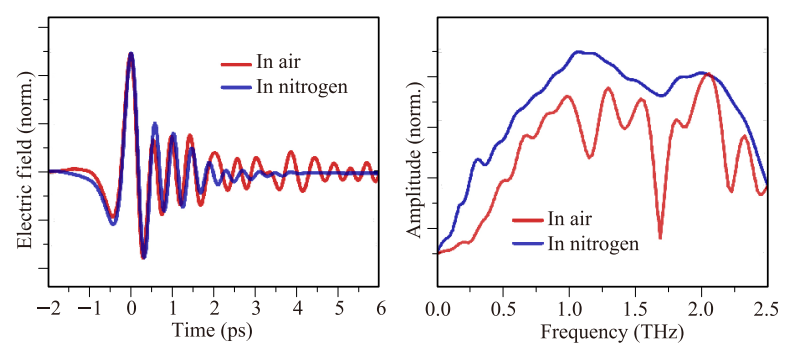

Fig. 3 Time-dependent electric field of $\mathrm{THz}$ probe pulse (left) and corresponding Fourier amplitude as a function of frequency (right) measured in air and nitrogen. could undergo subtle changes in direction/position during evacuation, the current measurement was performed by filling the chamber with nitrogen gas. Samples sit at the end of a cold finger in a helium continuous-flow cryostat, which is capable of reaching a base pressure as low as $2 \times 10^{-5} \mathrm{~Pa}$ and a temperature of $4 \mathrm{~K}$.

There are three time delays in this system, as shown in Fig. 1, which are set along the signal 2 path (time delay I), pump arm (time delay II), and $\mathrm{THz}$ detection arm (time delay III). Time delay I is used for precise compensation of the optical path differences between signal 1 and signal 2, which is a prerequisite for DFG. By moving time delay II while keeping time delay III at the peak position of the $\mathrm{THz}$ electric field, the pump-probe time delay, denoted as $\tau$, can be acquired. $\tau$ refers to the delay after excitation of the sample and reveals the changes in the peak of the $\mathrm{THz}$ electric field induced by a pump pulse. The transient changes in the time-dependent $\mathrm{THz}$ electric field at $\tau$ can be captured by moving both time delay II and time delay III in the same step to set the relative delay between the detection pulses and $\mathrm{THz}$ pulses while keeping the pump and detection pulses relatively still. The time delay between the sampling pulse and the $\mathrm{THz}$ pulse, $t$, maps out the $\mathrm{THz}$ spectrum at each $\tau$. The time resolution of this system is not determined by the envelope of the $\mathrm{THz}$ pulses, which is generally over 1 ps long, but by the Fourier limit of the pulse, i.e., the pulse width of $35 \mathrm{fs}$.

There are two choppers in this system, as shown in Fig. 1, which are set along the $\mathrm{THz}$ detection arm (chopper I) and the pump arm (chopper II). There are two methods of acquiring the transient changes in the $\mathrm{THz}$ electric field $\Delta E(t)$. In one case, the equilibrium reflected electric field $E_{0}(t)$ and the after-pumping field $E^{\prime}(t)$ are measured by modulation using chopper I, so $\Delta E(t)$ can be calculated as $\Delta E(t)=E^{\prime}(t)-E_{0}(t)$. In the other case, $\Delta E(t)$ is acquired directly by modulation of the pump pulse with chopper II by filtering the EOS signal with a lock-in amplifier. Typically, the results of these two methods should be identical, which was confirmed with our spectroscopy by the measurement of $\mathrm{EuSbTe}_{3}$ as shown below.

To evaluate the performance of our system, we performed $15 \mu \mathrm{m}$ pump and $1.2 \mu \mathrm{m}$ pump- $\mathrm{THz}$ probe measurements of a bulk EuSbTe ${ }_{3}$ crystal at room temperature.

The top left panel in Fig. 4 depicts the relaxation of the sample after excitation by $15 \mu \mathrm{m}$ pump pulses. Here pump scans are taken by fixing $\mathrm{THz}$ time delay III at the peak position of the $\mathrm{THz}$ electric field. The pump-induced changes in the reflected $\mathrm{THz}$ electric field $(\Delta E / E)$ acquired directly by modulation of the pump pulse with chopper II at each $\tau$ are shown in the top right panel in Fig. 4, in which the changes in the electric 

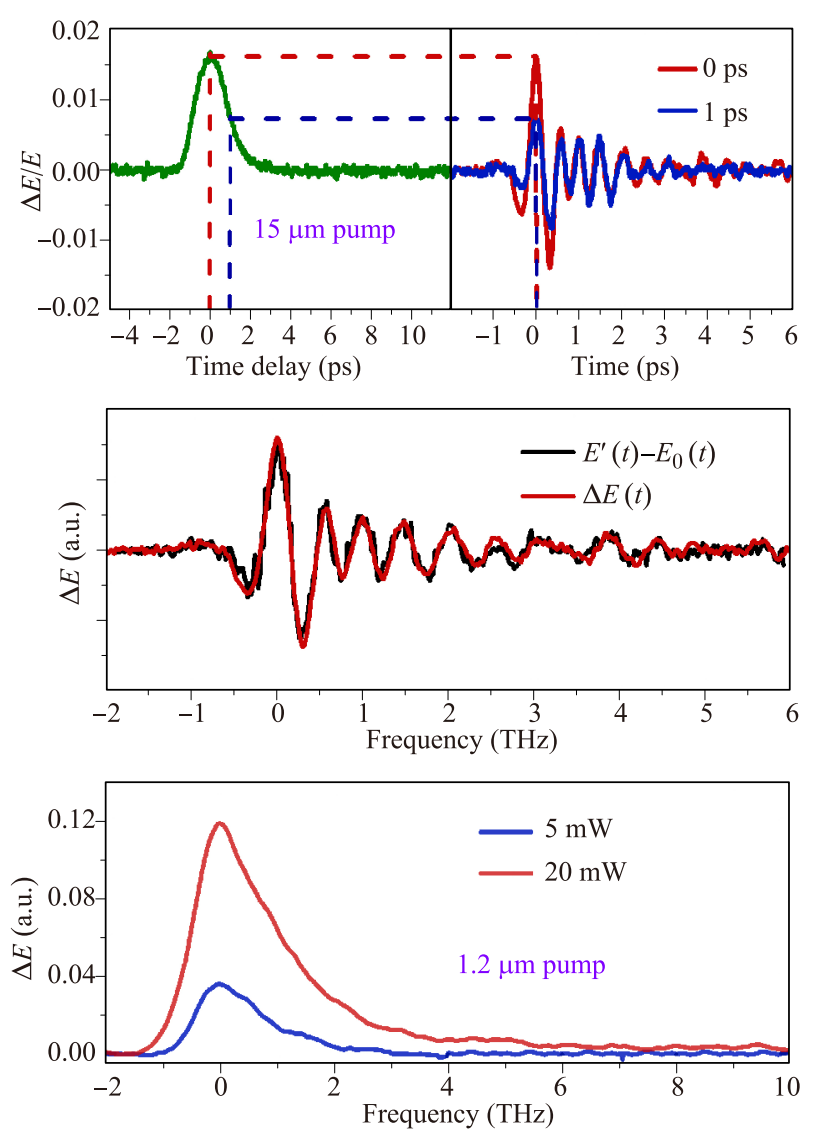

Fig. $415 \mu \mathrm{m}$ and $1.2 \mu \mathrm{m}$ pump-THz probe measurements of $\mathrm{EuSbTe}_{3}$ at room temperature. Top left: Relaxation of the sample after $15 \mu \mathrm{m}$ excitation. Top right: Pump-induced changes in the reflected $\mathrm{THz}$ electric field $(\Delta E / E)$ at each $\tau$. Center: Difference between the equilibrium reflected electric field $E_{0}(t)$ and the after-pumping field $E^{\prime}(t)$ at $\tau=0$ ps (red), and $\Delta E(t)$ measured directly by modulation of the pump (blue). Bottom: Relaxation of the sample after $1.2 \mu \mathrm{m}$ excitation.

fields match well with those observed in the pump-probe measurements (dashed lines are guides for the eyes.). A $0.5 \%$ transient change in the reflected $\mathrm{THz}$ electric field can be unambiguously measured in our experiment.

For $\mathrm{EuSbTe}_{3}$, the equilibrium reflected electric field $E_{0}(t)$ and the after-pumping field $E^{\prime}(t)$ at $\tau=0 \mathrm{ps}$ were also measured by modulation with chopper I, and $\Delta E(t)$ was calculated using the function $\Delta E(t)=E^{\prime}(t)-E_{0}(t)$. These two different methods are compared in the center panel of Fig. 4. It can be concluded that the obtained transient changes in the $\mathrm{THz}$ reflected electric field $(\Delta E / E)$ are identical regardless of which pump pulses or $\mathrm{THz}$ pulses are modulated. However, a much higher signal-to-noise ratio can be achieved by modulation of the pump pulse because the pump-induced changes in the field $\Delta E(t)$ are very small compared to $E_{0}(t)$. The $15 \mu \mathrm{m}$ pump- $\mathrm{THz}$ probe experimental mea- surement of bulk $\mathrm{EuSbTe}_{3}$ demonstrates that the constructed ultrafast broadband infrared pump- $\mathrm{THz}$ probe spectroscopy is credible and sensitive enough to observe subtle changes in the reflectivity configuration. It is expected to reveal novel phenomena in time-resolved $\mathrm{THz}$ spectroscopy experiments, in particular in modeselective control measurement of strongly correlated electron systems.

NIR pump-THz probe spectroscopy can be enabled by simply blocking one of the signal beams from the twooutput OPA and removing the germanium plate in the pump arm, as a slight displacement of the temporal and spatial overlaps of the pump and probe pulses can be easily corrected by moving time delay II and tuning the adjuster screws of the silver mirror in Fig. 1, respectively. In fact, NIR pump- $\mathrm{THz}$ probe spectroscopy is much easier to manipulate than MIR pump spectroscopy, as there is always a small amount of visible light in the NIR pulses that can be used for spatially overlapping the pump and THz-generating $800 \mathrm{~nm}$ pulses, i.e., overlapping the pump and $\mathrm{THz}$ probe pulses by direct visual observation (the subtle displacement between the $\mathrm{THz}$ pulses and the $800 \mathrm{~nm}$ pulses is negligible here). The bottom panel of Fig. 4 shows the relaxation of the sample after excitation by $1.2 \mu \mathrm{m}$ pump pulses with two different fluences. The pump-induced change in the $\mathrm{THz}$ electric field at each $\tau$ could be obtained as described for the $15 \mu \mathrm{m}$ pump case. This experimental apparatus can also be used for a $800 \mathrm{~nm}$ pump-THz probe experiment by compensating for the optical path differences. A $400 \mathrm{~nm}$ pump- $\mathrm{THz}$ probe experiment is feasible using a barium borate crystal.

To summarize, we constructed an ultrafast broadband infrared pump-THz probe spectroscopy system, which is capable of detecting subtle changes in the reflected electric field of the $\mathrm{THz}$ probe. A two-output OPA is used for generating MIR pulses with wavelengths of $15 \mu \mathrm{m}$ or beyond, where GaSe is used as the nonlinear crystal. This type of ultrafast broadband spectroscopy has been proven as a powerful tool for light control of different orders in strongly correlated materials, which is able to pumping low energy excitations in materials and probing the transient photoinduced changes with terahertz pulses. Room-temperature measurements of bulk EuSbTe $_{3}$ using a $15 \mu \mathrm{m}$ pump and $0.2-2.5 \mathrm{THz}$ probe were performed, and a $0.5 \%$ transient change in the reflected $\mathrm{THz}$ electric field was clearly detected with sufficient signal-to-noise ratio.

Acknowledgements We thank Dr. Dong Wu for supplying the $\mathrm{EuSbTe}_{3}$ single crystals that were used for the apparatus testing measurements. This work was supported by the National Science Foundation of China (Grant Nos. 11327806 and GZ1123) and the National Key Research and Development Program of China (Grant No. 2016YFA0300902). 
Open Access This article is distributed under the terms of the Creative Commons Attribution License which permits any use, distribution, and reproduction in any medium, provided the original author(s) and the source are credited.

\section{References}

1. S. G. Han, Z. V. Vardeny, K. S. Wong, O. G. Symko, and G. Koren, Femtosecond optical detection of quasiparticle dynamics in high- $T_{\mathrm{c}} \mathrm{YBa}_{2} \mathrm{Cu}_{3} \mathrm{O}_{7-\delta}$ superconducting thin films, Phys. Rev. Lett. 65(21), 2708 (1990)

2. G. L. Eesley, J. Heremans, M. S. Meyer, G. L. Doll, and S. H. Liou, Relaxation time of the order parameter in a high-temperature superconductor, Phys. Rev. Lett. 65(27), 3445 (1990)

3. S. Iwai, M. Ono, A. Maeda, H. Matsuzaki, H. Kishida, H. Okamoto, and Y. Tokura, Ultrafast optical switching to a metallic state by photoinduced Mott transition in a halogen-bridged nickel-chain compound, Phys. Rev. Lett. 91(5), 057401 (2003)

4. R. I. Tobey, S. Wall, M. Först, H. Bromberger, V. Khanna, J. J. Turner, W. Schlotter, M. Trigo, O. Krupin, W. S. Lee, Y. D. Chuang, R. Moore, A. L. Cavalieri, S. B. Wilkins, H. Zheng, J. F. Mitchell, S. S. Dhesi, A. Cavalleri, and J. P. Hill, Evolution of threedimensional correlations during the photoinduced melting of antiferromagnetic order in $\mathrm{La}_{0.5} \mathrm{Sr}_{1.5} \mathrm{MnO}_{4}$, Phys. Rev. B 86(6), 064425 (2012)

5. M. Porer, U. Leierseder, J. M. Ménard, H. Dachraoui, L. Mouchliadis, I. E. Perakis, U. Heinzmann, J. Demsar, K. Rossnagel, and R. Huber, Non-thermal separation of electronic and structural orders in a persisting charge density wave, Nat. Mater. 13(9), 857 (2014)

6. M. C. Hoffmann and J. A. Fülöp, Intense ultrashort terahertz pulses: Generation and applications, J. Phys. D Appl. Phys. 44(8), 083001 (2011)

7. D. Fausti, R. I. Tobey, N. Dean, S. Kaiser, A. Dienst, M. C. Hoffmann, S. Pyon, T. Takayama, H. Takagi, and A. Cavalleri, Light-induced superconductivity in a stripe-ordered cuprate, Science 331(6014), 189 (2011)

8. A. Dienst, E. Casandruc, D. Fausti, L. Zhang, M. Eckstein, M. Hoffmann, V. Khanna, N. Dean, M. Gensch, S. Winnerl, W. Seidel, S. Pyon, T. Takayama, H. Takagi, and A. Cavalleri, Optical excitation of Josephson plasma solitons in a cuprate superconductor, Nat. Mater. 12(6), 535 (2013)

9. W. Hu, S. Kaiser, D. Nicoletti, C. R. Hunt, I. Gierz, M. C. Hoffmann, M. Le Tacon, T. Loew, B. Keimer, and A. Cavalleri, Optically enhanced coherent transport in $\mathrm{YBa}_{2} \mathrm{Cu}_{3} \mathrm{O}_{6.5}$ by ultrafast redistribution of interlayer coupling, Nat. Mater. 13(7), 705 (2014)
10. D. Nicoletti and A. Cavalleri, Nonlinear light-matter interaction at terahertz frequencies, Adv. Opt. Photonics 8(3), 401 (2016)

11. D. N. Basov, R. D. Averitt, D. Van Der Marel, M. Dressel, and K. Haule, Electrodynamics of correlated electron materials, Rev. Mod. Phys. 83(2), 471 (2011)

12. S. Kaiser, C. R. Hunt, D. Nicoletti, W. Hu, I. Gierz, H. Y. Liu, M. Le Tacon, T. Loew, D. Haug, B. Keimer, and A. Cavalleri, Optically induced coherent transport far above $T_{\mathrm{c}}$ in underdoped $\mathrm{YBa}_{2} \mathrm{Cu}_{3} \mathrm{O}_{6+\delta}$, Phys. Rev. $B$ 89(18), 184516 (2014)

13. W. Shi, Y. J. Ding, N. Fernelius, and K. Vodopyanov, Efficient, tunable, and coherent $0.18-5.27 \mathrm{THz}$ source based on GaSe crystal, Opt. Lett. 27(16), 1454 (2002)

14. R. Huber, A. Brodschelm, F. Tauser, and A. Leitenstorfer, Generation and field-resolved detection of femtosecond electromagnetic pulses tunable up to $41 \mathrm{THz}, A p p l$. Phys. Lett. 76(22), 3191 (2000)

15. K. Reimann, R. Smith, A. Weiner, T. Elsaesser, and M. Woerner, Direct field-resolved detection of terahertz transients with amplitudes of megavolts per centimeter, Opt. Lett. 28(6), 471 (2003)

16. A. Sell, A. Leitenstorfer, and R. Huber, Phase-locked generation and field-resolved detection of widely tunable terahertz pulses with amplitudes exceeding $100 \mathrm{MV} / \mathrm{cm}$, Opt. Lett. 33(23), 2767 (2008)

17. K. Liu, J. Xu, and X. C. Zhang, GaSe crystals for broadband terahertz wave detection, Appl. Phys. Lett. 85(6), 863 (2004)

18. C. Kübler, R. Huber, S. Tübel, and A. Leitenstorfer, Ultrabroadband detection of multi-terahertz field transients with GaSe electro-optic sensors: Approaching the near infrared, Appl. Phys. Lett. 85(16), 3360 (2004)

19. A. Rice, Y. Jin, X. F. Ma, X. C. Zhang, D. Bliss, J. Larkin, and M. Alexander, Terahertz optical rectification from $\langle 110\rangle$ zinc-blende crystals, Appl. Phys. Lett. 64(11), 1324 (1994)

20. Q. Wu and X. C. Zhang, Free-space electro-optics sampling of mid-infrared pulses, Appl. Phys. Lett. 71(10), 1285 (1997)

21. Y. Y. Niu, D. Wu, L. Shen, and B. Wang, A layered antiferromagnetic semiconductor $\mathrm{EuMTe}_{3}(\mathrm{M}=\mathrm{Bi}, \mathrm{Sb})$, Phys. Status Solidi Rapid Res. Lett. 9(12), 735 (2015)

22. M. Van Exter, C. Fattinger, and D. Grischkowsky, Terahertz time-domain spectroscopy of water vapor, Opt. Lett. 14(20), 1128 (1989)

23. Y. Yang, A. Shutler, and D. Grischkowsky, Measurement of the transmission of the atmosphere from 0.2 to $2 \mathrm{THz}$, Opt. Express 19(9), 8830 (2011) 